\title{
19
}

\section{AN EFFICIENT FORM-BASED STRATEGY FOR THE RELIABILITY ANALYSIS OF MARINE STRUCTURES}

\author{
L. Manuel ${ }^{\mathrm{a}}$ and C.A.Cornell
}

Jack R. Benjamin \& Assoc., Inc., 444 Castro St., Suite 501, Mountain View, CA 94041

bept. of Civil Engineering, Stanford University, Stanford, CA 94305-4020.

\section{ABSTRACT}

In structural reliability analyses, one often finds that response computations needed to examine limit state(s) can be very expensive. Within a first- or second-order reliability method (FORM or SORM) framework which involves an iterative search for the design point, these limit state functions (and their gradients) need to be repeatedly evaluated. Clearly, it would be desirable to minimize the number of such evaluations while still retaining the same rate of convergence and the overall analysis framework.

Using a marine structure for illustration, we propose a FORM-based strategy that achieves this objective. It does so by making only approximate computations for gradients of the limit state function. These approximations are based on localized exact response estimates and we do not perform any additional response computations for them. The approximate gradients are computed by employing user-defined response transfer functions and response surfaces. The proposed strategy is shown to be efficient and accurate when compared to conventional FORM analyses.

\section{INTRODUCTION}

In most structural reliability applications, the pertinent random variables can be broadly divided into three classes: load, capacity, and response. Of these, response analyses required to establish the distribution of the random response are often the most expensive. For example, in the reliability of marine structures, load and capacity random variables may be thought to make up the long-term reliability problem. Dynamic response analyses conditional on load and capacity realizations form the short-term reliability problem and are the most expensive part of the overall reliability analysis, often requiring time-domain structural response simulations.

When FORM analyses are employed, inherent in the methodology is a need to iteratively evaluate the performance function and its gradients (with respect to all random variables) at each new point during the search for the design point. Because each such evaluation often involves expensive short- 
term response analyses, one would like to minimize the number of these evaluations but at the same time retain the general framework of the first-order method and not significantly degrade the efficiency of the search for the design point.

We propose a strategy which might be thought of as deviating only slightly from a conventional FORM approach: in each iteration, gradients of the limit state function are obtained by simpler approximate methods (not full-blown short-term response analyses). The precise nature of these approximations is intimately tied to aspects of the problem such as the importance of dynamics and the extent to which the response is non-Gaussian. We will show how fairly accurate gradients may be computed when gross (up to second order) statistics are captured by employing response transfer functions and higher order statistics of the response are captured by employing adaptive response surfaces.

The manner in which the marine structural reliability analysis problem is usually formulated, namely with a clear distinction between short-term (response) and long-term (load and capacity) random variables, makes this problem a good candidate for demonstration of our proposed strategy. The proposed strategy may, however, be generalized to include a broader class of problems than ones associated with marine structure reliability. Essentially, any reliability problem where limit state function evaluations can be expensive would benefit from a strategy such as the one proposed here where response transfer functions and response surfaces are employed and defined entirely within the FORM framework.

In our numerical studies, we will use a jack-up rig subjected to random hydrodynamic loading. We choose this structure because of its many interesting aspects. Jack-up rigs are dominated by nonGaussian drag loads and are flexible, and thus dynamically sensitive especially when used in deep water. Several recent studies have addressed issues dealing with the behavior and reliability of jackup rigs (see, for example, Karunakaran et al. [2], Kjeøy et al. [3], Løseth et al. [4], Manuel [5], McDonald and Bea [6]). We will study two different jack-ups and will use a long-term environmental description representative of the North Sea. We will define required response transfer functions and response surfaces (for our proposed strategy) in two different ways for each structure. We will compare our results with those obtained using the conventional FORM approach.

\section{FORMULATION}

\subsection{Conventional Approach}

The conventional first-order reliability method (FORM) algorithm, in its most common implementation, involves the following set of steps that need to be performed iteratively after defining a vector $\boldsymbol{X}$ made up of $N$ random variables, and a limit state function, $g(\boldsymbol{X})$ (see, for example, Rackwitz and Fiessler [7])

Step 1 Guess $\boldsymbol{x}_{0}$ (of size $N$ ) as an estimate of the (unknown) design point, $\boldsymbol{x}^{*}$

Step 2 Evaluate $g\left(x_{0}\right)$

Step 3 Obtain $N$ gradients of $g\left(x_{0}\right)$

Step 4 Improve estimate of $x^{*}$ using $g\left(x_{0}\right)$ and gradients; return to Step 1 with improved estimate of $x^{*}$ if no convergence yet.

It is immediately clear from the above algorithm that in Steps 2 and 3 above, a total of $N+1$ evaluations of the limit state function are required within each FORM iteration.

\subsection{Proposed Strategy and Modification to Conventional Approach}

In order to describe our proposed strategy, it is most appropriate to compare the steps in the approach we will take with those involved in the conventional algorithm. The essential differences 
are a direct result of our objective which is to minimize the number of limit state function evaluations per iteration (which, we have seen above, is $N+1$ in the conventional approach). Very simply stated, we will achieve this by doing away with Step 3 in its conventional sense. We will thus require only one conventional limit state function evaluation (for Step 2); however, we will evaluate the $N$ required gradients (for Step 3) only in an approximate manner taking advantage of information we have about the limit state function locally (from Step 2 of the present iteration) by defining response transfer functions there and by also building (and improving adaptively) response surfaces based on a specified number of preceding iterations in our search for the design point. Computational savings are a result of our use of "approximate" gradients which will not require response analyses of the same level of accuracy as we would employ in Step 2.

In order to describe our proposed strategy in detail, it is convenient to think of the random variable vector, $\boldsymbol{X}$, as being made up of two parts: a load/system variable vector, $\boldsymbol{W}$, and a response random variable, $Z$. Long-term joint distributions for $W$ will generally be available from environmental descriptions and structural system properties. The function of short-term response analyses is to establish the conditional distribution of $Z$ given $W$. The random variable $Z$ is usually a critical response measure (for example, a response extreme or a cumulative damage measure) of a time-varying underlying response process, $Y(t)$. Evaluations of the limit state function and its gradients implicitly involve establishing (in each iteration) the conditional distribution of $Z$ given $W$. When this conditional distribution is represented by a standard distribution type, this implies establishing the parameters of such a distribution.

Assume that a vector of parameters, $\boldsymbol{\theta}$, needs to be estimated in order to establish the conditional distribution of $Z$ given $W$ and evaluate the limit state function. Assume also, that in Step 2 of iteration $j$, we have estimated this parameter vector, $\boldsymbol{\theta}_{j}$. In Step 3 of the same iteration, we will then need to estimate $\theta_{j, \Delta i}$ in order to obtain the gradient in the direction $i$ (where $\theta_{j, \Delta i}$ is our shorthand notation for the parameter vector resulting from an incremental change in random variable $W_{i}$ in iteration $j$ ). It is to obtain $\boldsymbol{\theta}_{j, \Delta i}$ that we will employ our response transfer functions and response surfaces.

In general, a clear distinction will be possible among (1) the entire parameter vector, $\theta$, (2) those parameters, $\boldsymbol{\Omega}$, that retain gross statistical information (up to second order moments, i.e., r.m.s. level) and (3) other parameters, $\Psi$, that will have to capture any higher order statistics. In a problem such as the one chosen for illustration here (the reliability analysis of a jack-up rig to random wave loading), the role of the former sub-set, $\Omega$, might also be thought of as capturing the dynamic characteristics of the response while that of the latter sub-set, $\Psi$, might be thought of as preserving the non-Gaussian nature of the loading or capturing quasi-static characteristics of the response. With a defined distinction of this type, we have that $\theta=\{\Omega, \Psi\}$ and we will next describe procedures for establishing $\boldsymbol{\Omega}_{j, \Delta i}$ and $\boldsymbol{\Psi}_{j, \Delta i}$, the former using response transfer functions and the latter using response surfaces. This will, then, enable us to establish $\boldsymbol{\theta}_{j, \Delta i}$ and thus, gradients in iteration $j$.

\subsection{Establishing Response Transfer Functions for $\Omega$ in iteration $j$}

If the parameter vector, $\Omega$, is to include response statistics only up to second order, we can select suitable load measures, $L_{\mu}$ and $L_{\sigma}$, with respect to which we will define response transfer functions $R\left(\mu, \boldsymbol{w}_{j}\right)$ and $R\left(\sigma^{2}, \boldsymbol{w}_{j}, f\right)$ (in terms of realizations $\boldsymbol{w}_{j}$ of the random variable vector, $\boldsymbol{W}$ in Step 2 of iteration $j$, where the variable $f$ corresponds to frequency) that will enable computation (in Step 3 ) of the mean and standard deviation ( $\mu$ and $\sigma$ ) of the underlying response process, $Y(t)$ as follows:

$R\left(\mu, w_{j}\right)=\frac{E\left[Y\left(w_{j}\right)\right]}{E\left[L_{\mu}\left(w_{j}\right)\right]}$ 
$R\left(\sigma^{2}, w_{f}, f\right)=\frac{S_{Y}\left(w_{f}, f\right)}{S_{L_{0}}\left(w_{f}, f\right)}$

where $E\left[Y\left(w_{j}\right)\right]$ and $S_{Y}\left(\boldsymbol{w}_{j}, f\right)$ are the mean value and the power spectral density of $Y(t)$ as obtained in Step $2 ; E\left[L_{\mu}\left(\boldsymbol{w}_{j}\right)\right]$ and $S_{L \sigma}\left(\boldsymbol{w}_{j}, f\right)$ are the mean value and the power spectral density, respectively, of the two selected load measures. Note the dependence on the current values of the load/system variables $\boldsymbol{w}_{j}$, i.e., the system is presumed to be linear only locally and only for small deviations (gradients).

\subsection{Establishing Response Surface for $\Psi$ in iteration $j$}

In Step 2 of iteration $j$, the parameter vector $\Psi$ of size $P$ retained from the last $N_{f}$ iterations (up to and including the $j$ th iteration) is used to establish the matrix $\boldsymbol{A}$ required to define an adaptive response surface as follows:

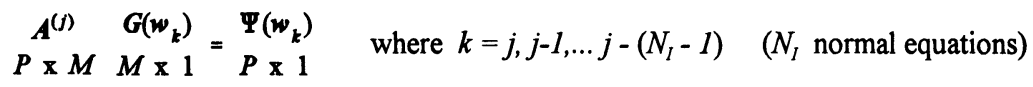

It is important to note that the user selects the number $N_{I}$ of iterations to be used in building the response surface. The user also selects the functional form of the vector $G(w)$ and the size $M$ of this vector. Regarding these selections, it is necessary that $N_{I} \geq M$; also, a judicious choice of $\boldsymbol{G}(\boldsymbol{w})$ (based on the user's preliminary judgement of a functional dependence of $\boldsymbol{\Psi}$ on $W$ ) can be important in improving the rate of convergence. This is especially true in early iterations. $N_{I}, M$ and the form of $G(w)$ can be changed as the search progresses; indeed, early in the process, $N_{I}$ can clearly not be large since there will not have been a sufficient number of iterations already carried out. Then, $M$ and $G(w)$ must be such that they permit simpler functional forms for $G(w)$ that are needed. In general, the procedure for building a response surface as defined above may either be based on a least squares minimization using the $N_{I}$ normal equations when $N_{I}>M$ or on collocation when $N_{I}=M$.

\subsection{Approximate Gradients in iteration $j$}

In order to evaluate gradients of the limit state function in iteration $j$, the parameter vector $\boldsymbol{\theta}_{j, \Delta i}$ must be defined for each random variable $W_{i}$. It is convenient to think of the limit state function in iteration $j$ as $\mathrm{g}\left(\boldsymbol{w}_{j}, Z\left(\boldsymbol{w}_{j}, \boldsymbol{\theta}_{j}\right)\right)$. Then, the gradient in the direction of $\boldsymbol{W}_{i}$ is given as follows:

$$
\left.\frac{\partial g}{\partial w_{i}}\right|_{X=x_{j}}=\frac{g\left(w_{j, \Delta i}, Z\left(w_{j, \Delta i}, \theta_{j, \Delta i}\right)\right)-g\left(w_{j}, Z\left(w_{j}, \theta_{j}\right)\right)}{\Delta w_{i}}
$$

where the unknown parameter vector, $\boldsymbol{\theta}_{j, \Delta i}=\left\{\boldsymbol{\Omega}_{j, \Delta i}, \boldsymbol{\Psi}_{j, \Delta i}\right\}$, can now be estimated as follows:

$$
\begin{aligned}
& \boldsymbol{\Omega}_{j, \Delta i}=\left[R\left(\mu, \boldsymbol{w}_{j}\right) \cdot E\left[L_{\mu}\left(\boldsymbol{w}_{j, \Delta i}\right)\right], \int_{0}^{\infty} R\left(\boldsymbol{\sigma}^{2}, \boldsymbol{w}_{j}, f\right) \cdot S_{L_{0}}\left(\boldsymbol{w}_{j, \Delta i}, f\right) d f\right] \\
& \boldsymbol{\Psi}_{j, \Delta i}=A^{(j)} G\left(\boldsymbol{w}_{j, \Delta i}\right)
\end{aligned}
$$

where $R\left(\mu, \boldsymbol{w}_{j}\right)$ and $R\left(\sigma^{2}, \boldsymbol{w}_{j}, f\right)$ are defined in Equation (1), $A^{(j)}$ is defined in Equation (2), and the incremental change in the direction of $W_{i}$ together with the definitions of $L_{\mu}, L_{\sigma}$, and $\boldsymbol{G}(\boldsymbol{w})$ make it possible to evaluate $\boldsymbol{\theta}_{j, \Delta i}$. This in turn enables us to compute $g\left(\boldsymbol{w}_{j, \Delta i}, Z\left(\boldsymbol{w}_{j, \Delta i}, \boldsymbol{\theta}_{j, \Delta i}\right)\right)$ and the desired gradients using Equation (3).

As can be seen from the above formulation of our proposed strategy, response analyses carried out in Step 2 are followed by additional computations in order to define "new" local response transfer 
functions and to "update" response surfaces. These additional computations are, however, extremely easy ones and make it unnecessary to perform $N$ additional response analyses in order to compute required gradients.

\section{NUMERICAL STUDIES}

We now address the reliability of a jack-up rig subjected to random wave loading. Additional non-random wind and current are also present. Salient aspects of the geometry, the material properties, and the loading are described in Figure 1.

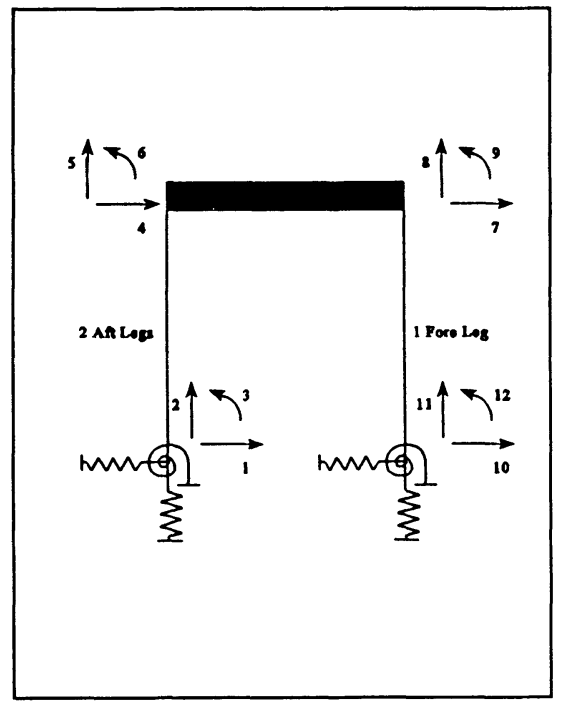

Wave Spectrum: JONSWAP $(\gamma=3.3)$

Depth of water, $\quad d=75.0 \mathrm{~m}$

Steady Wind Force, $F_{\text {wind }}=0.27 \times 10^{6} \mathrm{~N}$

Current, $u_{0}(z)=0.8 \mathrm{~m} / \mathrm{s}(-50 \mathrm{~m}<z<0 \mathrm{~m})$

$0.4 \mathrm{~m} / \mathrm{s}(-75 \mathrm{~m}<z<-50 \mathrm{~m})$

Length of each leg $\quad=100.0 \mathrm{~m}$

Spacing between fore and aft legs $=51.0 \mathrm{~m}$

Spacing between two aft legs $\quad=55.0 \mathrm{~m}$

Weight of each leg $\quad=1.03 \times 10^{7} \mathrm{~N}$

Weight of hull $\quad=8.53 \times 10^{7} \mathrm{~N}$

Leg Flexural Rigidity $\quad=1.56 \times 10^{12} \mathrm{~N}-\mathrm{m}^{2}$

Area of cross-section of each leg $=0.48 \mathrm{~m}^{2}$

P- $\delta$ effects included

Airy wave theory with Wheeler stretching

Relative Morison hydrodynamics

Structural damping: 2 percent of critical.

For pinned model: displacements 1-2, 10-11 $=0$

For fixed model: displacements $1-3,10-12=0$

Figure 1. Jack-up Rig Geometry, Material Properties, Loading, and Modeling Assumptions.

The prototype is a three-legged jack-up rig that is triangular hull in plan. We employ an idealized two-dimensional structural frame model to represent it. In this model, the dominant loading direction is assumed to be from aft to fore of the rig. The left leg of the frame model represents the two aft legs and the right leg represents the single fore leg of the prototype. Fixity conditions at the soilstructure interface will be varied in order to determine the reliability of jack-ups with different flexibilities; pinned and fixed end conditions will be compared.

In addition to the random wave loading, a steady current velocity profile collinear with the waves is adopted. A steady wind force also in the direction of the dominant wave loading is applied at the level of the deck. In order to obtain hydrodynamic forces due to waves and current, we employ Airy wave theory along with Wheeler stretching corrections. Full response analyses in our examples consist of time-domain load and response simulations necessary to obtain stable estimates of response extremes for the duration of a seastate.

Consider a first-excursion limit state function where $Y(t)$ represents the instantaneous deck displacement of our jack-up rig and $Y_{m}$ represents an extreme value of $Y(t)$ in a seastate of duration 
$T$ (in our examples, $T=3$ hours). We will assume that our random variable vector, $\boldsymbol{X}$, is made up of two load-related random variables (significant wave height, $H_{s}$, and spectral peak period, $T_{p}$ ) and one response random variable, $Y_{m}$. In our previous notation, $\boldsymbol{X}=\{\boldsymbol{W}, Z\}$ where $\{\boldsymbol{W}\}=\left\{H_{s}, T_{p}\right\}$ and $Z=Y_{m} . Y_{c}$ is a safe response threshold or a permissible deck displacement which we will assume to be deterministic. The limit state function may then be written as follows:

$g(W, Z)=Y_{c}-Y_{m}$

In the limit state function defined above, $Y_{m}$ is an implicit function of $W$. In our examples, the joint probability distribution $F_{w}(w)$ for the load random variables $H_{s}$ and $T_{p}$ and associated parameters are assumed to be those given by Bitner-Gregersen and Haver [1] for the Haltenbanken site off mid-Norway. The conditional distribution of $Z$ given $W$ must be an extreme value distribution type; we employ a Hermite transformation model (see Winterstein [7]) whose parameters may be shown to be related to the zero upcrossing rate, $v_{Y}(0)$, and the first four moments of the underlying deck displacement, $Y(t)$. Our vector of parameters, $\theta$, is needed to establish the conditional distribution, $F_{Z \mid W}(z)$. Only after this parameter vector is estimated, can the limit state function be evaluated. In our example studies, we have $\theta=\{\Omega, \Psi\}$ where $\Omega=\left\{\mu, \sigma^{2}\right\}$ and $\Psi=\left\{\alpha_{3}, \alpha_{4}, v_{7}(0)\right\}$ where $\alpha_{3}$ and $\alpha_{4}$ are the coefficients of skewness and kurtosis respectively of $Y(t)$.

For each jack-up structure (pinned and fixed), we will perform reliability analyses in three different ways: (1) using the conventional FORM approach, (2) using the quasi-static base shear $V$ to define our load measure of interest, i.e., $L_{\mu}=L_{\sigma}=V$, and (3) using $L_{\mu}=H_{s}$, the significant wave height, and $L_{\sigma}=\eta(t)$, the instantaneous sea surface elevation process. These approaches as listed are in decreasing order of effort required per FORM iteration. As discussed before, the conventional FORM approach involves $N+1$ response analyses (i.e., response simulations in the time domain) per iteration; the approach using base shear as the load measure of interest requires one full load and response simulation for Step 2 and $N$ additional load simulations for each random variable in order to obtain gradients for Step 3; finally, the approach using $L_{\mu}=H_{s}$ and $L_{\sigma}=\eta(t)$ only requires the single load and response simulation for Step 2 while for Step 3 neither load nor response simulations are required. In our example, $N_{I}=3$, and we choose $G(W)=\left\{1, T_{p}, H_{s}\right\}$, i.e., $N_{I}=M$ in Equation (2).

Jack-up rigs with two different fixity conditions are considered: (1) a pinned model where no rotational restraint is assumed at the bottom of each leg of the jack-up; the period of this structural model is about 5.6 seconds and hence, dynamic effects are fairly important in this case, and (2) a fixed model which is assumed to be fully restrained at the bottom of each leg; the period of this structure is about 2.8 seconds and, as a result, the behavior of the structure is quasi-static, and the response is highly non-Gaussian in this case.

The above two choices lead to contrasting dynamic and non-Gaussian features which, in turn, serve to highlight the importance of distinct aspects of our proposed strategy. In the pinned model where the dynamics are important, the response transfer functions play a vital role in the analysis; whereas, in the fixed model the very non-Gaussian nature of the response requires that the response surfaces capture higher order statistics (beyond a gross r.m.s. level) accurately.

In the following tables, we summarize the results obtained from all the reliability analyses. The two faster approximate methods produce virtually the same answers as the conventional approach without degradation of the search efficiency. 


\section{(1) Jack-up Pinned at Base}

Start at $H_{s}=2.38 \mathrm{~m}, T_{p}=9.23 \mathrm{~s}, Y_{m}=0.17 \mathrm{~m}$ (in standard normal space, $\left.\boldsymbol{u}_{0}=\{0,0,0\}\right)$ $g(X)=Y_{c}-Y_{m} ; Y_{c}=1.00 \mathrm{~m}$

\begin{tabular}{|c|c|c|c|c|c|c|c|c|c|}
\hline Method & $\begin{array}{r}\mathrm{De} \\
H_{s}(\mathrm{~m})\end{array}$ & $\begin{array}{l}\text { ign Poir } \\
T_{p}(\mathrm{~s})\end{array}$ & $\begin{array}{l}Y_{m}(\mathrm{~m}) \\
Y^{*}\end{array}$ & $\begin{array}{l}\text { Impo } \\
\alpha_{H s}^{2}\end{array}$ & $\begin{array}{c}\operatorname{tance} \\
\alpha^{2} \\
T_{p}\end{array}$ & $\begin{array}{r}\text { actors } \\
\alpha_{Y m}^{2}\end{array}$ & $\beta$ & $P_{f}$ & $\begin{array}{c}\text { Number } \\
\text { of Itera- } \\
\text { tions }\end{array}$ \\
\hline Conventional & 10.15 & 13.46 & 1.00 & 80.1 & 17.4 & 2.5 & 3.32 & $4.54 \times 10^{-4}$ & 14 \\
\hline $\begin{array}{l}\text { Approximation } \\
\text { using base } \\
\text { shear }\end{array}$ & 10.07 & 13.18 & 1.00 & 77.3 & 20.6 & 2.1 & 3.35 & $3.99 \times 10^{-4}$ & 13 \\
\hline $\begin{array}{l}\text { Approximation } \\
\text { using sea } \\
\text { surface elev. }\end{array}$ & 10.20 & 13.77 & 1.00 & 84.4 & 12.5 & 3.1 & 3.25 & $5.82 \times 10^{-4}$ & 13 \\
\hline
\end{tabular}

\section{(2) Jack-up Fixed at Base}

Start at $H_{s}=6.89 \mathrm{~m}, T_{p}=12.67 \mathrm{~s}, Y_{m}=0.95 \mathrm{~m}$ (in standard normal space, $\boldsymbol{u}_{0}=\{2,0,0\}$ ) $g(X)=Y_{c}-Y_{m} ; Y_{c}=0.25 \mathrm{~m}$

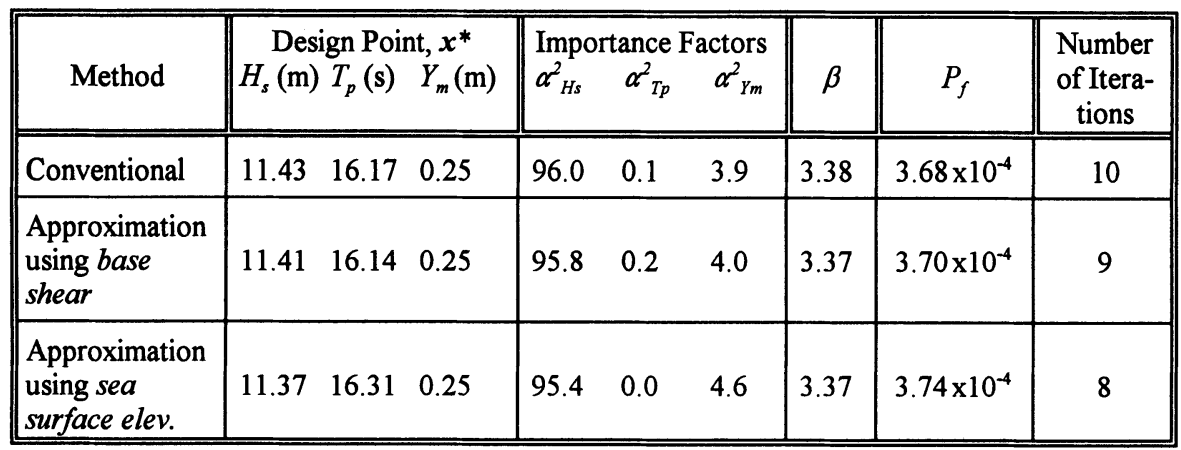

\section{DISCUSSION AND CONCLUSIONS}

A FORM-based strategy that uses response transfer functions and response surfaces has been employed to analyze two different jack-up rigs. Both base shear and sea surface elevations were found to perform adequately as load measures from which to infer locally linear response transfer functions.

The building of a response surface within the FORM search is adaptive (i.e., it requires no additional effort a priori). In the examples, a simple linear functional form was employed for the 
response surface and only the three preceding iterations were utilized to update the response surface in a given FORM iteration.

The contrasting nature of the structures studied revealed that in situations where the dynamics are significant (as in the response of the pinned jack-up model), the response transfer function definitions employed in the proposed strategy are very important. On the other hand, when the response is very non-Gaussian (as with the fixed jack-up model), the response surface definitions prove to be very important.

It was found that roughly the same number of iterations are required with the proposed strategy as with the conventional FORM method. However, the cost per iteration for the proposed strategy can be significantly lower. In the examples studied, we employed two different approximate strategies where the base shear and the sea surface elevation, respectively, were defined as important load measures. The base shear approximation resulted in 40 percent savings in cost per iteration while the sea surface elevation approximation resulted in 67 percent savings relative to the conventional FORM approach. If a larger number of random variables are involved, the savings realized from use of the proposed strategy can be expected to be far greater.

\section{ACKNOWLEDGMENTS}

The authors gratefully acknowledge the support of the following sponsors of the Reliability of Marine Structures (RMS) program at Stanford University: Amoco, Chevron, Conoco, DNVI, Exxon, Mobil, Norsk Hydro, Saga, Shell, Statoil, and Texaco.

\section{REFERENCES}

1. E.M.Bitner-Grigersen and S.Haver, Joint Long Term Description of Environmental Parameters for Structural Response Calculation, Proc. 12th Intl. Workshop on Wave Hindcasting and Forecasting, Vancouver, Canada, April 1989.

2. D.Karunakaran, B.J.Leira, S.Haver, and T.Moan, Parametric Influence of Extreme Dynamic Response of Drag-Dominated Platforms, Proc. Second Intl. Offshore and Polar Eng. Conf., Vol III, pp. 463-471, San Francisco, California, June 1992.

3. H.Kjeøy, N.G.Bøe, and T.Hysing, Extreme Response Analysis of Jack-Up Platforms, Marine Structures, 2:305-334, 1989.

4. R.Løseth, O.Mo, and I.Lotsberg, Probabilistic Analysis of a Jack-Up Platform with respect to the Ultimate Limit State, Proc. 1st European Offshore Mechanics Symp., pp. 322-330, Trondheim, Norway, August 1990.

5. L.Manuel, A Study of the Nonlinearities, Dynamics, and Reliability of a Drag-Dominated Marine Structure, Tech. Report RMS-12, Stanford Univ., Reliability of Marine Structures Program, Stanford, California, December 1992.

6. D.McDonald and R.G.Bea, Reliability Evaluation of a Jack-Up Drilling Unit, Trans. Soc. of Naval Architects and Marine Engineers, Annual Meeting, pp. 169-186, San Francisco, California, October 1990.

7. R.Rackwitz and B.Fiessler, Structural Reliability under Combined Random Load Sequences, Computers and Structures, 9:489-494, 1978.

8. S.R.Winterstein, Nonlinear Vibration Models for Extremes and Fatigue, J. of Eng. Mech., ASCE, 114(10), pp. 1772-1790, October 1988. 